Article

\title{
Investigation of the Interaction between Benzene and SXFA Using DFT
}

\author{
Katherine M. E. Stewart ${ }^{1, *,+}$, Ian P. Hamilton ${ }^{2}$ and Alexander Penlidis ${ }^{1}$ (I) \\ 1 Department of Chemical Engineering, Institute for Polymer Research, University of Waterloo, \\ Waterloo, ON N2L 3G1, Canada; penlidis@uwaterloo.ca \\ 2 Department of Chemistry, Wilfrid Laurier University, Waterloo, ON N2L 3C5, Canada; ihamilton@wlu.ca \\ * Correspondence: kastewart@troy.edu \\ $\dagger$ Current address: Department of Chemistry and Physics, Troy University, Troy, Al 36082, USA.
}

Received: 4 December 2017; Accepted: 22 January 2018; Published: 25 January 2018

\begin{abstract}
Density Functional Theory (DFT) studies were conducted to evaluate the sensing mechanism between benzene and a polymeric sensing material, referred to as SXFA, which contains trifluoro-groups and $\mathrm{OH}$-groups. These studies were undertaken to improve the understanding of how benzene and SXFA mechanistically interact based on their chemistry, information which can be used to more efficiently design polymeric sensing materials. We find that benzene adsorbed onto the $\mathrm{OH}$-groups in SXFA rather than the trifluoro-groups as previously proposed. Specifically, we find that sorption results from electrostatic attraction between the negative benzene ring and the positive hydrogens of the OH-groups of SXFA.
\end{abstract}

Keywords: benzene; Density Functional Theory (DFT); sensing mechanisms; gas sensors; polymeric sensing material

\section{Introduction}

Benzene is a byproduct of industrial processes and vehicle exhaust [1]. Benzene is toxic and contributes to poor indoor air quality, with no safe level recommended by the World Health Organization (WHO) [2]. Due to the high toxicity of benzene, various polymeric sensing materials have been developed to monitor benzene levels in air [3-6].

Polymers are ideal sensing materials because they operate at low temperatures (below $100{ }^{\circ} \mathrm{C}$ ) and are relatively inexpensive [6]. In addition, polymers can be tailor-made to attract a specific (target) gas, such as benzene, and thus, can have high selectivity [7]. This is done by modifying the polymer functional groups and side chains, thereby changing their surface chemistry so that they preferentially attract and adsorb a target analyte for a specific application.

To more efficiently design and tailor polymeric sensing materials for target analytes and specific applications, it is important to understand the sensing mechanisms by which analytes (and interferents) interact with sensing materials [8]. One way to investigate these sensing mechanisms is with Density Functional Theory (DFT) calculations [9,10].

Sensing mechanisms for a variety of gas sensors have recently been evaluated using DFT analysis [11-13]. These calculations have improved the understanding of how sensing materials and analytes interact with one another. By combining DFT studies with experimental work, sensing mechanisms can be better understood, which can significantly aid in improving the design of new sensing materials and sensors.

Recent experimental studies have shown that benzene preferentially adsorbs onto the trifluoro-containing polymer, SXFA (see Figure 1a) [14,15]. Chen et al. [14,15] evaluated SXFA at room temperature $\left(22{ }^{\circ} \mathrm{C}\right)$ on three different capacitive, radio frequency identification (RFID) 
sensing platforms, using a specially designed test system [16]. In all cases, SXFA was exposed to gaseous benzene, resulting in benzene adsorbing onto the sensing material, causing a measurable frequency shift on the RFID sensors (see Figure 1b), with sensitivities (per percent frequency shift) of $5.92 \times 10^{-4} / \%, 5.05 \times 10^{-4} / \%$, and $9.1 \times 10^{-5} / \%$ for RFID 1, RFID 2, and RFID 3, respectively [14,15].

(a)

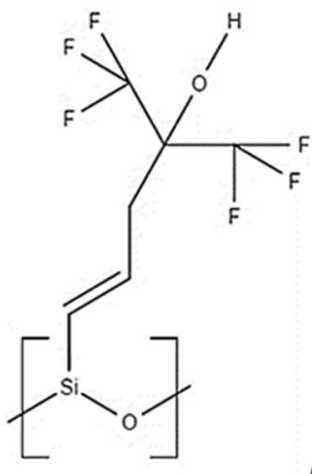

(b)

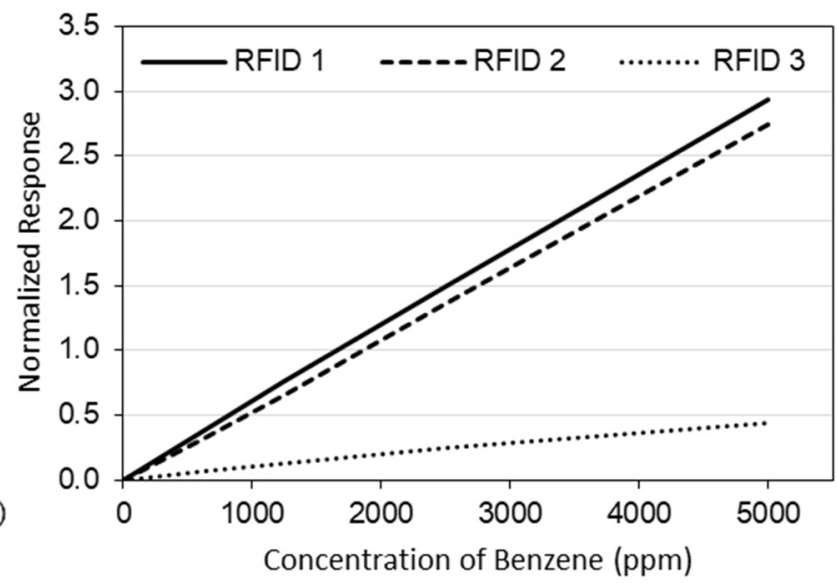

Figure 1. (a) Chemical structure of SXFA and (b) Normalized response to benzene of three different RFID sensors using SXFA as a sensing material [14,15] (RFID: radio frequency identification).

A sensing mechanism was subsequently suggested in which benzene $\pi$-stacks onto one of the trifluoro-groups on SXFA, since the fluorine atoms form a planar ring (see Figure 2) [8]. This paper evaluates the proposed sensing mechanism between benzene and SXFA using a DFT approach.
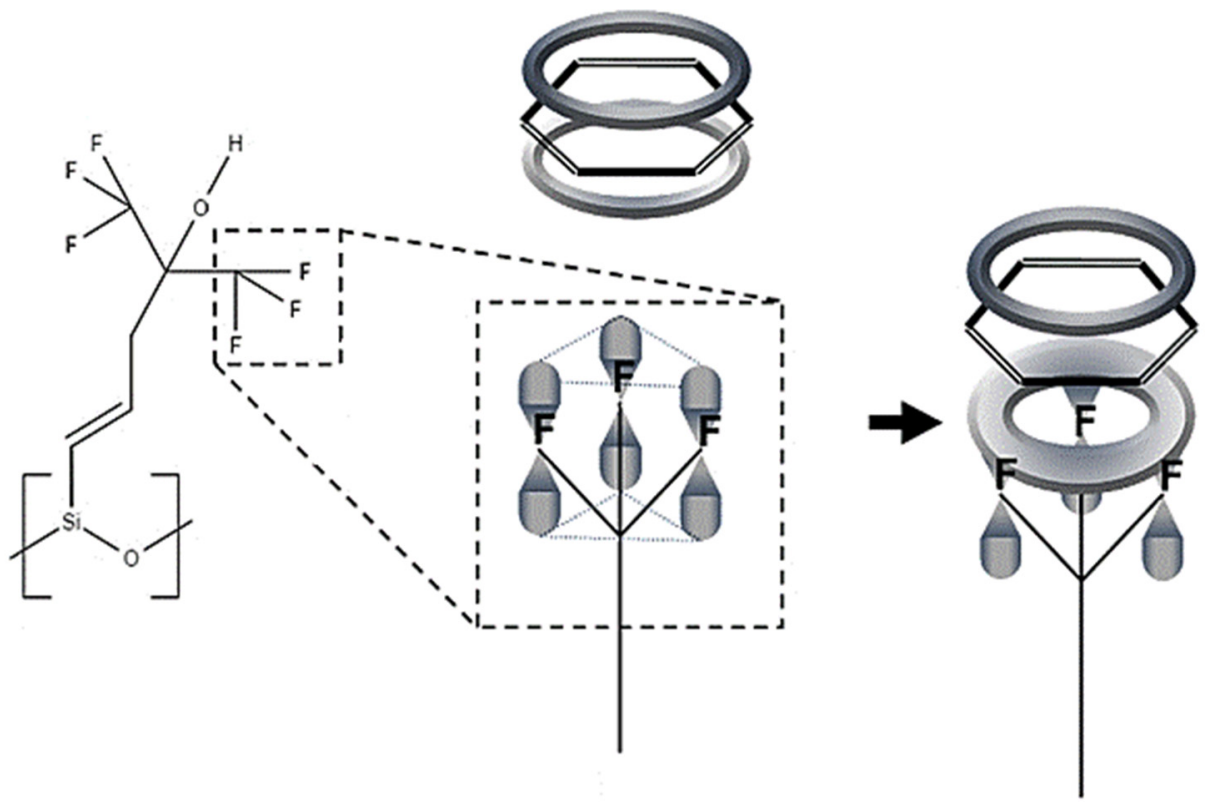

Figure 2. Proposed sensing mechanism of benzene and SXFA [8].

Previous studies have evaluated carbon tetrafluoride and benzene complexes both experimentally and computationally $[17,18]$. The optimized configuration of carbon tetrafluoride and benzene showed three fluorine atoms directed towards the benzene molecule. While this orientation of the carbon tetrafluoride-benzene complex agrees with the proposed sorption of benzene to SXFA, it has been shown that this orientation may not be explained by $\pi$-stacking, but rather charge transfer between the small molecules that reduces the electron density in the $\pi$-system [17]. 


\section{Methodology}

Calculations were performed using DFT with the B3LYP and M06 functionals and the LANL2DZ basis set, as implemented in Gaussian. The geometries were optimized from a variety of initial structures and complexes to ensure that the optimized geometry was the global minimum and not a function of the initial position of the molecules and atoms. Mulliken and Natural Bond Orbital (NBO) charges were used in the evaluation of the proposed sensing mechanism.

\section{Results and Discussion}

Initially, the complex of a single SXFA unit (a carbon chain with two trifluoro-groups and an OH-group) with benzene was evaluated. From there, short SXFA chains were evaluated, adding one SXFA unit at a time, up to four SXFA units. In all cases, and for both the B3LYP and M06 functionals, the benzene molecule oriented itself perpendicularly to the $\mathrm{OH}$-group (see Figure 3 ) and not parallel to the trifluoro-group, as postulated by Stewart and Penlidis [8].

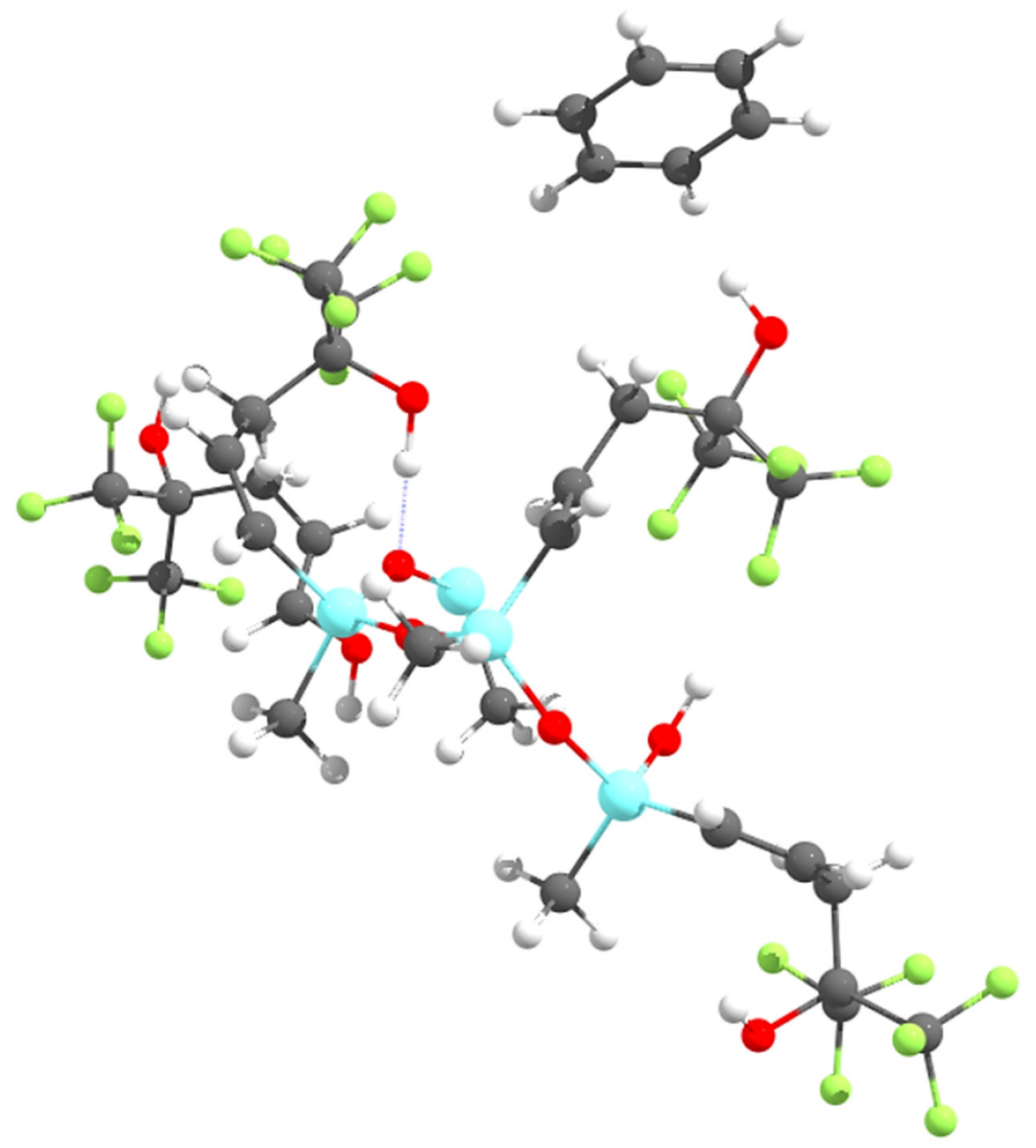

Figure 3. Benzene coordinating with the OH-group on SXFA.

Since the benzene molecule is not parallel to the trifluoro-group on SXFA, benzene does not sorb to the trifluoro-groups in SXFA. Instead, benzene preferentially sorbs to the OH-group in SXFA. Due to benzene's preference to interact with the $\mathrm{OH}$-group instead of either of the trifluoro-groups, it can be concluded that the sensing mechanism proposed by Stewart and Penlidis [8] is incorrect. Rather than benzene $\pi$-stacking with one of the trifluoro-groups on SXFA, benzene is attracted to the OH-group in SXFA, as shown in Figure 3.

To examine the nature of this attraction, the Mulliken and NBO atomic charges were calculated on all the atoms in benzene and SXFA. Figure 4 shows the Mulliken charges on each atom of the SXFA unit 
and benzene. Both the Mulliken and NBO atomic charges do not significantly change when additional SXFA units are added to the SXFA chain (see Table 1).

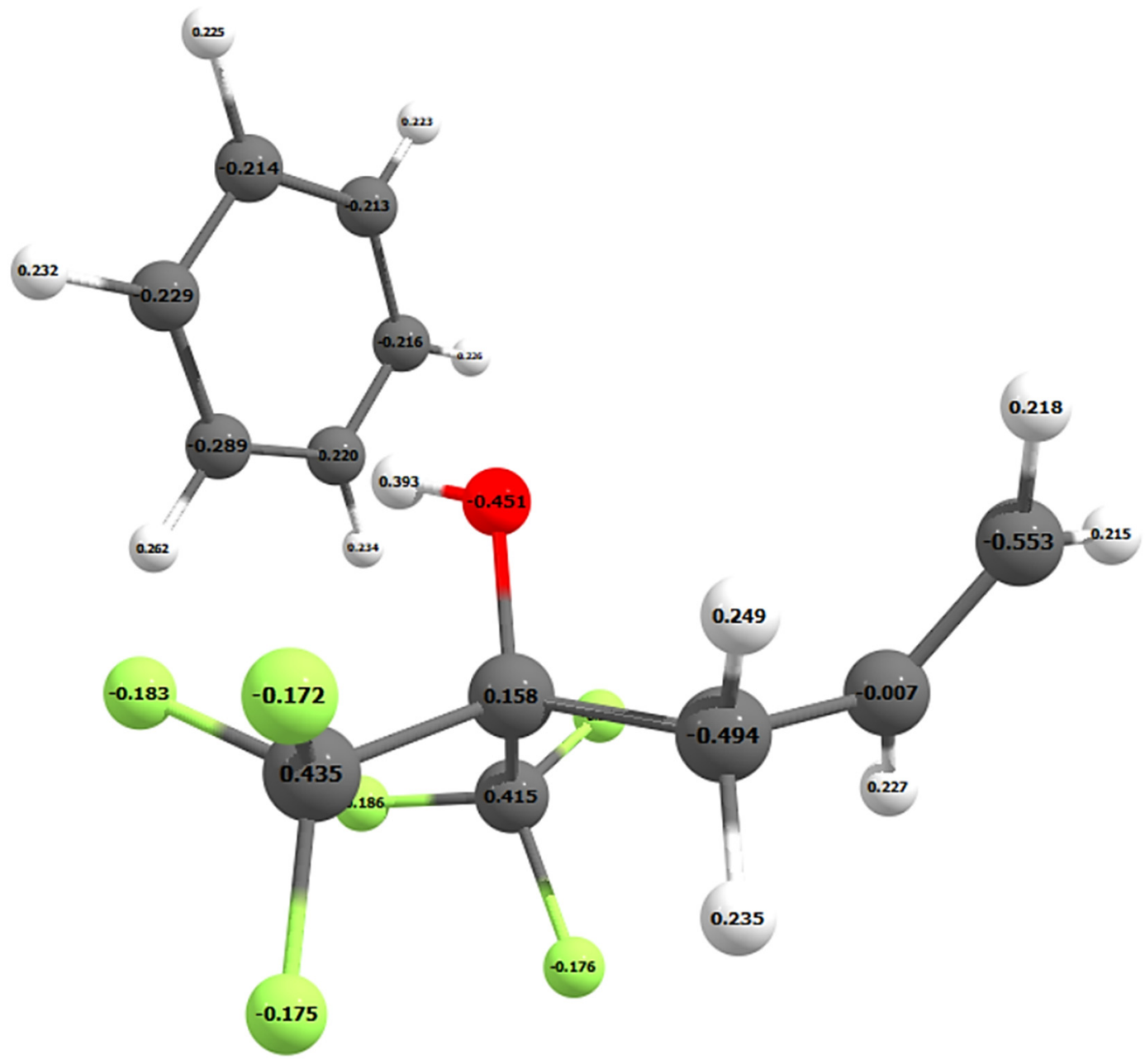

Figure 4. Mulliken atomic charges on the SXFA unit and benzene atoms.

Table 1. Mulliken and NBO Atomic Charges on OH-group.

\begin{tabular}{ccccc}
\hline Chain Length & $\begin{array}{c}\text { Mulliken Atomic } \\
\text { Charge on } \mathbf{~}\end{array}$ & $\begin{array}{c}\text { Mulliken Atomic } \\
\text { Charge on H }\end{array}$ & $\begin{array}{c}\text { NBO Atomic } \\
\text { Charge on O }\end{array}$ & $\begin{array}{c}\text { NBO Atomic } \\
\text { Charge on H }\end{array}$ \\
\hline 1 (Side chain only) & -0.451 & 0.393 & -0.785 & 0.529 \\
\hline 2 & -0.447 & 0.388 & -0.779 & 0.518 \\
& $-0.483^{*}$ & $0.459^{*}$ & $-0.781^{*}$ & 0.525 \\
\hline 3 & -0.448 & 0.387 & -0.777 & 0.521 \\
& -0.452 & 0.381 & -0.781 & 0.524 \\
& $-0.486^{*}$ & $0.454^{*}$ & $-0.812^{*}$ & $0.544^{*}$ \\
\hline \multirow{2}{*}{4} & -0.453 & 0.389 & -0.765 & 0.512 \\
& -0.454 & 0.380 & -0.781 & 0.524 \\
& -0.468 & 0.404 & -0.807 & 0.492 \\
& $-0.497^{*}$ & $0.468^{*}$ & $-0.811^{*}$ & $0.495^{*}$ \\
\hline
\end{tabular}

${ }^{*}$ Hydrogen bonded to the OH-group at the end of the Si-O chain.

Although the NBO analysis resulted in larger charges on the oxygen and hydrogen atoms, both the Mulliken and NBO charges show the same trend. Both DFT analyses calculated negative charges 
on the carbon atoms of the benzene ring and resulted in benzene orienting itself perpendicular to the $\mathrm{OH}$-group on SXFA (Figures 3 and 4). Therefore, benzene adsorbs onto the OH-group of SXFA and not one of the trifluoro-groups.

Delocalization of the electrons on a benzene ring, which draws electron density from the hydrogen atoms, results in benzene having a larger electron density in the middle of the ring. This results in benzene being more negative in the center of the ring [19]. Given that oxygen draws electron density away from the hydrogen in an $\mathrm{OH}$-group, this results in an electrostatic interaction between the positive hydrogen of the $\mathrm{OH}$-group on SXFA and the negative benzene ring, which can be seen in the positive and negative Mulliken atomic charges in Figure 4.

The bond length and angle of the OH-group on the SXFA chain were also evaluated (see Table 2). Note that the central SXFA unit was used in a chain with three repeating units for this comparison. When benzene was adsorbed, the $\mathrm{C}-\mathrm{O}-\mathrm{H}$ bond angle and the $\mathrm{O}-\mathrm{H}$ bond length both increased which can be attributed the electrostatic interaction noted above.

Table 2. Bond Length and Valence Angles of the OH-group.

\begin{tabular}{ccc}
\hline Bond Lengths and Angles & SXFA & Adsorbed Benzene \\
\hline C-O Length & $1.44438 \AA$ & $1.43784 \AA$ \\
O-H Length & $0.98162 \AA$ & $0.98789 \AA$ \\
C-O-H Angle & $111.294^{\circ}$ & $112.839^{\circ}$ \\
\hline
\end{tabular}

The electrostatic attraction between the hydrogen of the $\mathrm{OH}$-group and the benzene ring is consistent with other model studies which have examined the bonding of various small molecules, including water [20], alcohols (methanol and ethanol) [10], and ammonia [21], hydrogen bonding to the benzene ring. In addition, the benzene ring has been shown to interact electrostatically with cations through cation- $\pi$ interactions, both in the gaseous phase and in solution [22].

\section{Conclusions}

DFT calculations were used to determine the optimized geometry of the benzene-SXFA complex. It was found that benzene adsorbed onto the OH-group of SXFA rather than onto one of the trifluoro-groups, as had been proposed by Stewart and Penlidis [8]. We have found that the sorption of benzene onto SXFA is due to electrostatic attraction between benzene and the OH-group, where the negative charge in the benzene ring is attracted to the positive charge on the hydrogen in the OH-group.

This paper demonstrates that DFT analysis can be used to improve understanding of how analytes sorb onto sensing materials. This improved understanding of the interactions between sensing materials and analytes (molecules) at an atomic level can aid in the optimization of sensors for particular molecules.

Acknowledgments: The authors are grateful for financial support from the Natural Sciences and Engineering Research Council (NSERC) of Canada and the Canada Research (CRC) program. The authors would also like to acknowledge Compute Canada for their computing resources.

Author Contributions: K.M.E.S. performed the calculations, data interpretations, and wrote the paper. I.P.H. provided access to the computing resources from Compute Canada and revised several drafts of the paper. A.P. supervised the work and revised several drafts of the paper.

Conflicts of Interest: The authors declare no conflict of interest.

\section{References}

1. Lee, S.C.; Chiu, M.Y.; Ho, K.F.; Zou, S.C.; Wang, X. Volatile organic compounds (VOCs) in urban atmosphere of Hong Kong. Chemosphere 2002, 48, 375-382. [CrossRef]

2. World Health Organization. Exposure to Benzene: A Major Public Health Concern; Public Health and Environment, World Health Organization: Geneva, Switzerland, 2010. 
3. Mondal, R.K.; Dubey, K.A.; Bhardwaj, Y.K.; Varshney, L. Novel hybrid nanocarbons/poly (dimethylsiloxane) composites based chemiresistors for real time detection of hazardous aromatic hydrocarbons. Carbon 2016, 100, 42-51. [CrossRef]

4. Spinelle, L.; Gerboles, M.; Kok, G.; Persijn, S.; Sauerwald, T. Review of portable and low-cost sensors for the ambient air monitoring of benzene and other volatile organic compounds. Sensors 2017, 17, 1520. [CrossRef] [PubMed]

5. Lonergan, M.C.; Severin, E.J.; Doleman, B.J.; Beaber, S.A.; Grubbs, R.H.; Lewis, N.S. Array-based vapor sensing using chemically sensitive, carbon black-polymer resistors. Chem. Mater. 1996, 8, 2298-2312. [CrossRef]

6. Mabrook, M.; Hawkins, P. A rapidly-responding sensor for benzene, methanol, and ethanol vapours based on films of titanium dioxide dispersed in a polymer operating at room temperature. Sens. Actuators B 2001, 75, 197-202. [CrossRef]

7. Talwar, V.; Singh, O.; Singh, R.C. ZnO assisted polyaniline nanofibers and its application as ammonia gas sensor. Sens. Actuators B 2014, 191, 276-282. [CrossRef]

8. Stewart, K.M.E.; Penlidis, A. Designing polymeric sensing materials: What are we doing wrong? Polym. Adv. Technol. 2007, 28, 319-344. [CrossRef]

9. Avramov, P.V.; Kudin, K.N.; Scuseria, G.E. Single wall carbon nanotubes density of states: Comparison of experiment and theory. Chem. Phys. Lett. 2003, 370, 597-601. [CrossRef]

10. Tolmachev, A.M.; Firsov, D.A.; Kuznetsova, T.A.; Anuchin, K.M. DFT modeling of the adsorption of benzene, methanol, and ethanol molecules in activated carbon nanopores. Mol. Supramol. Struct. Interfaces 2009, 45, 177-183. [CrossRef]

11. Pannopard, P.; Khongpracha, P.; Probst, M.; Limtrakul, J. Gas sensing properties of platinum derivatives of single-walled carbon nanotubes: A DFT analysis. J. Mol. Graph. Model. 2009, 28, 62-69. [CrossRef] [PubMed]

12. Ullah, H.; Shah, A.A.; Bilal, S.; Ayab, K. DFT Study of Polyaniline $\mathrm{NH}_{3}, \mathrm{CO}_{2}$, and $\mathrm{CO}$ Gas Sensors: Comparison with Recent Experimental Data. J. Phys. Chem. C 2013, 117, 23701-23711. [CrossRef]

13. Tran, H.T.; Spencer, M.J. Zinc oxide for gas sensing of formaldehyde: Density functional theory modelling of the effect of nanostructure morphology and gas concentration on the chemisorption reaction. Mater. Chem. Phys. 2017, 193, 274-284. [CrossRef]

14. Chen, W.T.; Stewart, K.M.E.; Yang, C.K.; Mansour, R.R.; Carroll, J.; Penlidis, A. Wearable RF Sensor Array Implementing Coupling-Matrix Readout Extraction Technique. IEEE Trans. Microw. Theory Tech. 2015, 63, 4157-4168. [CrossRef]

15. Chen, W.T.; Stewart, K.M.E.; Mansour, R.R.; Penlidis, A. Novel underloaded radio-frequency (RF) resonant sensor for gaseous ethanol and interferents detection. Sens. Actuators A 2015, 230, 63-73. [CrossRef]

16. Stewart, K.M.E.; Penlidis, A. Novel test system for gas sensing materials and sensors. Macromol. Symp. 2013, 324, 11-18. [CrossRef]

17. Lazzaroni, R.; Calderone, A.; Bredas, J.; Rabe, J. Electronic structure of molecular van der Waals complexes with benzene: Implications for the contrast in scanning tunneling microscopy of molecular adsorbates on graphite. J. Chem. Phys. 1997, 107, 99-105. [CrossRef]

18. Menapace, J.; Bernstein, E. Van der Waals modes of solute/solvent clusters: Benzene-methane, -deuteriomethane, and -carbon tetrafluoride. J. Phys. Chem. 1987, 91, 2843-2848. [CrossRef]

19. Lee, E.; Kim, D.; Jurecka, P.; Tarakeshwar, P.; Hobza, P.; Kim, K. Understanding of assembly phenomena by aromatic-aromatic interactions: Benzene dimer and the substituted systems. J. Phys. Chem. A 2007, 111, 3446-3457. [CrossRef] [PubMed]

20. Suzuki, S.; Green, P.G.; Bumgarner, R.E.; Dasgupta, S.; Goddard, W.A., III; Blake, G.A. Benzene forms hydrogen bonds with water. Science 1992, 257, 942-945. [CrossRef] [PubMed]

21. Wanna, J.; Menapace, J.; Bernstein, E. Hydrogen bonded and non-hydrogen bonded van der Waals clusters: Comparison between clusters of pyrazine, pyrimidine, and benzene with various solvents. J. Chem. Phys. 1986, 85, 1795-1805. [CrossRef]

22. Dougherty, D.A. Cation-pi interactions in chemistry and biology: A new view of benzene, Phe, Tyr, and Trp. Science 1996, 271, 163-168. [CrossRef] [PubMed]

(C) 2018 by the authors. Licensee MDPI, Basel, Switzerland. This article is an open access article distributed under the terms and conditions of the Creative Commons Attribution (CC BY) license (http://creativecommons.org/licenses/by/4.0/). 